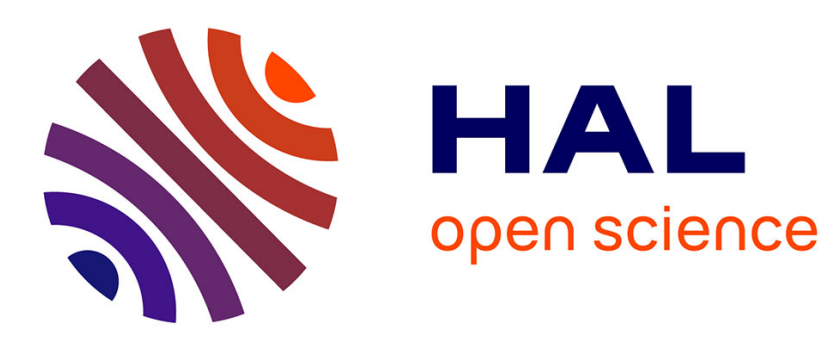

\title{
Localization of sparse and coherent sources by orthogonal least squares
}

Gilles Chardon, François Ollivier, José Picheral

\section{To cite this version:}

Gilles Chardon, François Ollivier, José Picheral. Localization of sparse and coherent sources by orthogonal least squares. Journal of the Acoustical Society of America, 2019, 146 (6), pp.4873-4882. 10.1121/1.5138931 . hal-02448965

\section{HAL Id: hal-02448965 \\ https://hal-centralesupelec.archives-ouvertes.fr/hal-02448965}

Submitted on 29 Jan 2020

HAL is a multi-disciplinary open access archive for the deposit and dissemination of scientific research documents, whether they are published or not. The documents may come from teaching and research institutions in France or abroad, or from public or private research centers.
L'archive ouverte pluridisciplinaire HAL, est destinée au dépôt et à la diffusion de documents scientifiques de niveau recherche, publiés ou non, émanant des établissements d'enseignement et de recherche français ou étrangers, des laboratoires publics ou privés. 


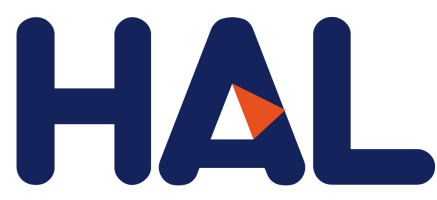

archives-ouvertes

\section{Localization of sparse and coherent sources by orthogonal least squares}

Gilles Chardon, François Ollivier, José Picheral

\section{To cite this version:}

Gilles Chardon, François Ollivier, José Picheral. Localization of sparse and coherent sources by orthogonal least squares. Journal of the Acoustical Society of America, Acoustical Society of America, 2019, 146 (6), pp.4873-4882. 10.1121/1.5138931 . hal-02448965

\section{HAL Id: hal-02448965 \\ https://hal-centralesupelec.archives-ouvertes.fr/hal-02448965}

Submitted on 29 Jan 2020

HAL is a multi-disciplinary open access archive for the deposit and dissemination of scientific research documents, whether they are published or not. The documents may come from teaching and research institutions in France or abroad, or from public or private research centers.
L'archive ouverte pluridisciplinaire HAL, est destinée au dépôt et à la diffusion de documents scientifiques de niveau recherche, publiés ou non, émanant des établissements d'enseignement et de recherche français ou étrangers, des laboratoires publics ou privés. 


\title{
Localization of sparse and coherent sources by Orthogonal Least Squares
}

\author{
Gilles Chardon, ${ }^{1}$ François Ollivier, ${ }^{2}$ and José Picheral ${ }^{1}$ \\ ${ }^{1)}$ L2S, CentraleSupelec - Université Paris Sud-CNRS, Université ParisSaclay, 2 rue Joliot Curie, \\ 91190 Gif sur Yvette, France \\ ${ }^{2}$ Sorbonne Université, CNRS, Institut Jean Le Rond d'Alembert, UMR 7190, F-75005 Paris, France
}

This paper proposes an efficient method for the joint localization of sources and estimation of their mutual correlations. In practice, such an estimation is useful to study correlated sources existing for instance in presence of reverberations or for spatially distributed sources, but is confronted to the challenge of computational complexity due to a large number of required estimates. The proposed method is named CMF-OLS (for Covariance Matrix Fitting by Orthogonal Least Squares). It is based on a greedy approach exploiting the OLS algorithm in order to reduce the computational complexity of the estimation. Compared to existing methods for sources correlation matrix estimation, its lower computational complexity allows to deal with large dimensions and to explore large regions of interest. As shown by numerical results, it is more accurate and does not require the tuning of any regularization parameter. Experiments involving reverberations show the ability of the method to locate and identify physical and mirror sources as well.

$$
\text { [http://dx.doi.org(DOI number)] }
$$

Pages: ??-??

\section{INTRODUCTION}

Source imaging methods have been developed for decades in order to locate acoustic sources and to estimate their power. Many works have proposed to overcome the well-known limitations of the delay and sum (DAS) beamforming technique, based on parametric approaches(?), or non-parametric approaches based on Bayesian estimation (??), or based on sparsity and compressed sensing (???).

This paper addresses the inverse problem of estimating the spatial covariance matrix of acoustic sources. This estimate not only quantifies the power of each point on the source grid but also estimates the correlation between each of these points.

In practice, sources correlation estimation can be useful in various scenarios: in a reverberant space, mirror sources will be correlated with the corresponding physical source. Estimating the coherence between sources will allow to pair physical sources with their reflections. Another typical case of correlated sources are extended sources whose distributions radiate correlated signals.

Localizing coherent sources poses an important challenge to subspace based localization methods such as MUSIC or ESPRIT. In this case the covariance matrix of the sources, and thus of the data, is rank-deficient, limiting the performances of these methods. For particular array configurations, this issue can be mitigated by the use of spatial smoothing, at the price of reduced array aperture (?). Methods based on sparse approximations

a) gilles.chardon@centralesupelec.fr or Bayesian estimation can deal with correlated sources, using the complete measurements (??).

In the cases where correlations are not seen as a nuisance, but as parameters to be estimated, an additional challenge is the computational complexity of the estimation. Compared to a standard localization problem, where the space is usually discretized on a grid of $L$ points, the estimation of the correlations between sources involves the estimation of a covariance matrix of size $L \times L$. The computational cost increases dramatically with the number of points requiring suitable algorithms. Numerical methods with a complexity larger than linear in the size of the grid will be limited to small grid discretizations.

Beyond the coarse estimation provided by the delay and sum beamformer extended to covariance estimation (called DAS-C in the remainder of the article), few methods have been proposed in the literature to solve the covariance source estimation problem (a short review is given in section II). Based on convex optimization methods, their complexity is in the order of $O\left(L^{6}\right)(?)$, or $O\left(L^{3} L_{h}^{3}\right)$ where $L_{h}$ is an upper bound on the number of independent sources (?). Such computational complexities will limit the application of these methods to coarse discretizations of the physical space.

In this paper, we propose a method named CMFOLS (for Covariance Matrix Fitting by Orthogonal Least Squares) to solve the covariance matching problem with a greedy approach. It is based on the Orthogonal Least Squares (OLS) algorithm (?), similar to the well-known Orthogonal Matching Pursuit algorithm (?), but better suited to the problem of covariance matrix estimation. Indeed, it can take full advantage of the matrix structure 
of the estimation problem. Moreover, OLS is expected to exhibit better behavior than OMP in cases where the dictionary is strongly coherent (in the sense that columns of the dictionary are near linearly dependent) (?).

The main advantage of this approach compared to existing methods is that its time complexity is linear with respect to the size of the discretization $L$, allowing fine grid discretizations and short running times. Moreover, in contrary to methods based on regularization, the algorithm takes only one parameter, the number of sources, that can be estimated by inspecting the convergence of the algorithm. A post-processing can then be applied to the estimated covariance matrix to extract the spatial shapes of each coherent source.

The article is structured as follows: Section ?? introduces the problem and notations. Correlated source localization methods and greedy sparse approximations are briefly reviewed in section ??. Section ?? and ?? introduce the CMF-OLS algorithm for covariance matrix estimation, and the post-processing respectively.

Simulation results presented in Section ?? performed in a $1 \mathrm{D}$ scenario show that the proposed method is more accurate and faster than the methods of the literature such as DAS-C or MACS. In addition, Section ?? introduces experiments that have been conducted in an anechoic room in various settings : uncorrelated sources, correlated sources, and reflections. Results confirm that positions and correlations of the sources can be accurately estimated in large-scale (in fact, realistic) scenarios where other methods are too demanding in time or memory size. Section ?? concludes the paper.

\section{PROBLEM FORMULATION}

Assume that $N$ microphones provides the $N \times 1$ measured signals $x\left(t_{i}\right)$ and let be $I$ the number of available snapshots.

The possible positions of the source are described by the parameter $\Theta \in \mathcal{R}$. The region $\mathcal{R}$ can be, depending on the cases, a line, a surface, or a volume, and is discretized on a grid of $L$ points defined by their position $\left\{\Theta_{\ell}\right\}_{\ell=1, \ldots, L}$.

Assuming that the discretization of $\mathcal{R}$ is fine enough, sources can be considered located at a point of the grid. For narrow band sources, the measured signals can be modeled by:

$$
\mathbf{x}\left(t_{i}\right)=\sum_{\ell=1}^{L} \mathbf{a}\left(\Theta_{\ell}\right) s_{\ell}\left(t_{i}\right)+\mathbf{n}\left(t_{i}\right)
$$

where $\mathbf{a}_{l}=\mathbf{a}\left(\Theta_{\ell}\right)$ is the $N \times 1$ steering vector for a source at the position $\Theta_{\ell}, s_{\ell}\left(t_{i}\right)$ is the source amplitude at the instant $t_{i}$ and $\mathbf{n}\left(t_{i}\right)$ is an additive centered noise assumed to be spatially white of variance $\sigma^{2}$. The coefficients of the steering vector $\mathbf{a}\left(\Theta_{\ell}\right)$ are the values of the Green function of the propagation medium between the position parametrized by $\Theta_{l}$ and a microphone at position $\mathbf{r}_{n}$. In an homogeneous medium and assuming free propagation, the Green function is $G\left(\mathbf{r}_{1}, \mathbf{r}_{2}\right)=\exp \left(-j\left\|\mathbf{r}_{1}-\mathbf{r}_{2}\right\|\right) / \| \mathbf{r}_{1}-$ $\mathbf{r}_{2} \|$.

Let the $N \times L$ matrix $\mathbf{A}=\left[\mathbf{a}\left(\Theta_{1}\right), \ldots, \mathbf{a}\left(\Theta_{L}\right)\right]$ be formed by the $L$ steering vectors associated to the $L$ points of the source grid, the signal model can be rewritten as

$$
\mathbf{x}\left(t_{i}\right)=\mathbf{A s}\left(t_{i}\right)+\left(t_{i}\right),
$$

where the $L \times 1$ vector $\mathbf{s}\left(t_{i}\right)=\left[s_{1}\left(t_{i}\right), \ldots, s_{L}\left(t_{i}\right)\right]$ contains the amplitudes of the sources.

Under the hypothesis of independence between the source signals and the noise, the covariance matrix can be expressed as:

$$
\boldsymbol{\Gamma}=E\left\{\mathbf{x}\left(t_{i}\right) \mathbf{x}\left(t_{i}\right)^{H}\right\}=\mathbf{A C A}^{H}+\sigma^{2} \mathbf{I},
$$

where $\mathbf{C}=E\left\{\mathbf{s}\left(t_{i}\right) \mathbf{s}\left(t_{i}\right)^{H}\right\}$ is the $L \times L$ source covariance matrix (or spatial covariance matrix). Estimation of $\mathbf{C}$ is the key point of the work presented in this paper since its diagonal describes the power of the sources, and its offdiagonal coefficients the covariance between each source.

In practice, the covariance matrix $\boldsymbol{\Gamma}$ is estimated by the sample covariance matrix using the set of $I$ measurements:

$$
\mathbf{G}=\frac{1}{I} \sum_{i=1}^{I} \mathbf{x}\left(t_{i}\right) \mathbf{x}\left(t_{i}\right)^{H} \approx \mathbf{\Gamma}
$$

The objective of our method will be to estimate the positions, powers, and mutual covariances of the sources from the matrix $\mathbf{G}$.

\section{STATE OF THE ART}

\section{A. Correlated sources estimation}

A first estimation of the source covariance matrix is given by the delay-and-sum beamformer for covariance estimation (DAS-C):

$$
\widehat{\mathbf{C}}_{D A S-C}=\frac{1}{L^{2}} \mathbf{A}^{H} \mathbf{G} \mathbf{A} \text {. }
$$

The diagonal of $\widehat{\mathbf{C}}_{D A S-C}$ is the output of the standard DAS beamformer, and has the same limitations, i.e. wide main lobes at low frequencies and presence of sidelobes.

In the case of punctual sources, the sparsity of the covariance matrix can be promoted by using an componentwise $\ell_{1}$ regularization term, as in the Sparse Spectrum Fitting method (??). The covariance matrix is estimated as the solution of the following optimization problem:

$$
\begin{array}{r}
\widehat{\mathbf{C}}_{S p S F}=\underset{\mathbf{C}}{\operatorname{argmin}}\left\|\mathbf{G}-\mathbf{A C A}^{H}\right\|_{F}^{2}+\lambda\|\mathbf{C}\|_{1} \\
\text { s.t. } \quad \mathbf{C} \geq 0 .
\end{array}
$$

Alternatively, the CMF-C method (?) jointly estimates the covariance matrix and the noise level assuming that the rank of the covariance matrix is low. The estimate is found as the solution of an optimization algorithm fitting the covariance matrix to the data while 
bounding its trace, equal to the $\ell_{1}$ norm of its eigenvalues, as a convex surrogate for limiting its rank:

$$
\begin{aligned}
& \widehat{\mathbf{C}}_{C M F-C}=\underset{\mathbf{C}, \sigma}{\operatorname{argmin}}\left\|\mathbf{G}-\mathbf{A C A}^{H}-\sigma^{2} \mathbf{I}\right\|_{F}^{2}, \\
& \text { s.t. } \quad \mathbf{C} \geq 0, \quad \operatorname{tr}(\mathbf{C}) \leq \lambda, \quad \sigma^{2} \geq 0 .
\end{aligned}
$$

Sparsity and low-rankedness can be combined using two regularization terms (?), yielding the following penalized optimization problem:

$$
\begin{array}{r}
\widehat{\mathbf{C}}_{S P L R}=\underset{\mathbf{C}}{\operatorname{argmin}}\left\|\mathbf{G}-\mathbf{A C A}^{H}\right\|_{F}^{2}+\lambda_{1}\|\mathbf{C}\|_{1}+\lambda_{2} \operatorname{tr}(\mathbf{C}), \\
\text { s.t. } \quad \mathbf{C} \geq 0 .
\end{array}
$$

These three optimisation problems are convex, and can be solved using off-the-shelf toolboxes such as CVX (??), or more specialized algorithms (e.g. Simultaneous Directions of Multipliers Method).

However, the computational complexity of solving these algorithms prevents their application for large-scale problems, where the discretization grid has e.g. more than $\approx 1000$ elements. Estimation of the covariance matrix by Sparse Spectrum Fitting and CMF-C with standard convex optimization $\left(O\left(L^{6}\right)\right)$ methods, is therefore limited to low dimensional problems, i.e. coarse discretization of the physical space.

Yardibi et al. proposed MACS (Mapping of Acoustic Correlated Sources) (?) with reduced complexity by assuming that the rank of the covariance matrix $\mathbf{C}$ to be estimated is at most $L_{h}$, an upper bound for the number of independent sources. The covariance matrix is factorized as $\mathbf{C}=\tilde{\mathbf{C}} \tilde{\mathbf{C}}^{H}$, where $\tilde{\mathbf{C}}$ has dimension $L \times L_{h}$. By adding an additional sparsity constraint on the coefficients of $\tilde{\mathbf{C}}$, the following non-convex problem is formulated:

$$
\underset{\mathbf{C}, \mathbf{Q}}{\operatorname{argmin}}\left\|\overline{\mathbf{G}} \mathbf{Q}^{H}-\mathbf{A C}\right\|_{F}^{2} \quad \text { s.t. } \quad\|\mathbf{C}\|_{1} \leq \beta, \quad \mathbf{Q}^{H} \mathbf{Q}=\mathbf{I}
$$

where $\overline{\mathbf{G}}$ is the matrix square root of a low-rank approximation of $\mathbf{G}$. The authors propose an algorithm alternating between $\ell_{1}$ constrained least-squares minimization and updates of $\mathbf{Q}$, which is not guaranteed to converge towards a global minimum. Improved MACS (IMACS) (?) was recently proposed, by updating the bound $\beta$ between the iterations.

The complexity of the estimation is reduced to $O\left(L^{3} L_{h}^{3}\right)$, which makes estimation problems of moderate dimensions tractable. However, the complexity remains too high for large scale or real time problems, as running time of the order of the minute are announced for problems of moderate dimensions $(L<1000)$.

\section{B. Greedy source localization}

An alternative to regularization based sparse estimation methods are greedy algorithms. They are known to be computationally less expensive than optimization based method, at the price of reduced performances. In particular, Orthogonal Matching Pursuit (OMP) (?) has been used for several acoustical inverse problems, such as Nearfield Acoustical Holography (?), source localization (?), or DAMAS-like deconvolution of beamformer output $(?)$.

In general, OMP aims at estimating a sparse solution of the undetermined linear system $\mathbf{y}=\mathbf{D} \mathbf{x}$, where $\mathbf{y}$ are the observations, $\mathbf{D}$ a known dictionary with normalized columns $\mathbf{d}_{i}$ (called atoms), and $\mathbf{x}$ the sparse coefficients to be estimated. OMP works as follows:

1. Initialization: $k=1$, residual $\mathbf{r}_{0}=\mathbf{y}$, set of identified indices $S_{0}=\emptyset$

2. Computation of the correlations of the residual with the dictionary, $\rho_{k, l}=\left|\mathbf{d}_{l}^{H} \mathbf{r}_{k-1}\right|^{2}$

3. An atom (i.e. a nonzero coefficient in $\mathbf{x}$ ) is identified by the maximal correlation $l^{\star}=$ $\operatorname{argmax}_{1 \leq l \leq L} \rho_{k, l}$

4. Its index is added to the set $S_{k+1}=S_{k} \cup\left\{l^{\star}\right\}$

5. The residual is updated by projecting $\mathbf{y}$ on the orthogonal of the space spanned by identified atoms: $\mathbf{r}_{k}=\mathbf{y}-\Pi_{S_{k}}(\mathbf{y})$

6. $k=k+1$ and go to step 2 until a stopping criterion is met (number of iterations, norm of the residual, etc.).

Under certain conditions on the dictionary $\mathbf{D}$ and the number of non-zero coefficients in $\mathbf{x}$, OMP is guaranteed to recover $\mathbf{x}$ exactly. Variants of OMP can deal with structured sparsity, e.g. block sparsity (?), where the support of $\mathbf{x}$ has a specific structure. In particular, block sparsity can be applied to joint localization and characterization of anisotropic sources (?) or extended sources (??).

\section{ORTHOGONAL LEAST SQUARES FOR CORRELATED SOURCES LOCALIZATION}

In this section, a greedy method is proposed for correlated sources localization, using a slightly different algorithm, Orthogonal Least Squares. The use of this algorithm is justified by its better behavior in presence of correlated dictionaries (?), which is the case in source localization, when the possible source positions are discretized with a step smaller than the main lobe width. Moreover, the selection criterion of OLS can be efficiently computed using the matrix structure of the problem.

For a set of indices $S$, we name $\mathbf{A}_{S}$ the matrix with columns $\mathbf{a}_{l}, l \in S$ extracted from $\mathbf{A}$.

The goal of sparse correlated sources estimation is to decompose the data matrix $\mathbf{G}$ as

$$
\mathbf{G} \approx \mathbf{A}_{\tilde{S}} \tilde{\mathbf{C}} \mathbf{A}_{\tilde{S}}^{H}
$$

where $\tilde{S}$ is the set of indices of the $J$ sources, and the positive definite matrix $\tilde{\mathbf{C}}$ is the $J \times J$ covariance matrix of the sources. In particular, the power of the individual 
sources can be found on its diagonal. The data matrix can be rewritten

$$
\mathbf{G} \approx \sum_{i \in \tilde{S}} \sum_{j \in \tilde{S}} \mathbf{a}_{i} \mathbf{a}_{j}^{H} \tilde{\mathbf{C}}_{i j}
$$

showing that $\mathbf{G}$ is a linear combination of $J^{2}$ rank-1 matrices $\mathbf{a}_{i} \mathbf{a}_{j}^{H}$.

With the complete dictionary A of steering vectors, $\mathbf{G}$ can be decomposed as:

$$
\mathbf{G} \approx \mathbf{A C A}^{H}
$$

where $\mathbf{C}$ is positive semi-definite and sparse, as it has $J^{2}$ nonzero coefficients, corresponding to the $J$ sources and their mutual covariances. These nonzero coefficients are given by the coefficients of $\tilde{\mathbf{C}}$.

Standard greedy recovery algorithm, such as OMP, can be used to estimate $\mathbf{C}$ using the following reformulation of the problem:

- The matrix $\mathbf{G}$ is vectorized as the $N^{2} \times 1$ vector $\mathbf{g}$

- A dictionary of rank-one matrices $\mathbf{D}_{1}$ is built, of dimension $N^{2} \times L^{2}$, with vectorized rank-1 matrices $\mathbf{a}_{l} \mathbf{a}_{m}^{H}$ as its columns.

- The covariance matrix $\mathbf{C}$ is vectorized as $\mathbf{c}$, with dimension $L^{2} \times 1$.

This reparametrization yields the linear problem $\mathbf{g}=$ $\mathbf{D}_{1} \mathbf{c}$. The direct application of OMP to this problem is however computationally expensive $\left(\mathbf{D}_{1}\right.$ has dimension $N^{2} \times L^{2}$, and $J^{2}$ iterations are necessary), and the particular structure of the dictionary (a collection of rank1 matrices) and of the decomposition coefficients (they form a positive definite matrix) are not used.

\section{A. Leveraging the structure}

The estimation of the covariance matrix $\mathbf{C}$ can be improved using structured sparsity, i.e. the particular structure of its support. Here, the structure of the support of the covariance matrix $\mathbf{C}$ stems from its non-negativity.

Indeed, in addition to the equality $\mathbf{C}_{l m}=\overline{\mathbf{C}}_{m l}$, the absolute value of the off-diagonal terms is controlled by the diagonal terms: $\left|\mathbf{C}_{l m}\right|=\left|\mathbf{C}_{m l}\right| \leq \sqrt{\mathbf{C}_{l l} \mathbf{C}_{m m}}$. This implies that off-diagonal terms appear as pairs, and only if the associated diagonal terms are non-zero. It is then sufficient to know the support of the diagonal terms to control the support of the complete matrix.

An efficient sparse recovery algorithm will thus identify a diagonal term at each iteration $k$, along with the $2(k-1)$ off-diagonal terms describing the covariance of this source with the previously identified sources. This selection rule implies that only $J$ iterations are needed. The principle of the selection rule is illustrated on figure ??. At step 1, a first source is identified. At step 2, a second source and its covariance with the first source are identified, etc. until all sources are located.
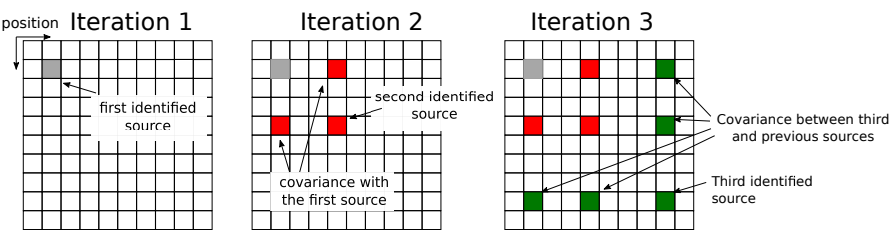

FIG. 1. Principle of the greedy source identification algorithm: at each step, a source (diagonal term) is identified along the covariance between itself and the previously identified sources.

The OMP algorithm can be modified to take into account structured sparsity. In particular, block structures can be identified by replacing the scalar product in step 3, by orthogonal projection on the space spanned by the atoms of a block (?). In the covariance matrix case, a block is a diagonal term along with its $2(k-1)$ associated off-diagonal terms. Step 3 of OMP is replaced by:

- For all $l$, compute the norms of the projection of the residual matrix $\mathbf{R}_{k-1}$ on the space $E_{k, l}$ spanned by $\mathbf{a}_{m} \mathbf{a}_{l}^{H}, \mathbf{a}_{l} \mathbf{a}_{m}^{H}$ and $\mathbf{a}_{l} \mathbf{a}_{l}^{H}$ for $m \in S_{k-1}: \rho_{k, l}=$ $\left\|\Pi_{E_{k, l}} \mathbf{R}_{k-1}\right\|_{2}^{2}$.

The matrix structure can be efficiently leveraged by using the Orthogonal Least-Squares algorithm. OLS is similar to OMP, with the identification step replaced by the maximization of the projection of the signal to be decomposed in the space spanned by the candidate atoms and the previously identified atoms. Step 3 of the OMP algorithm is further replaced by the following step:

- compute the norms of the projection of the residual on the space $F_{k, l}$ spanned by $\mathbf{a}_{n} \mathbf{a}_{m}^{H}$, for $n, m \in$ $S_{k, l}=\left(S_{k-1} \cup\{l\}\right): \rho_{k, l}=\left\|\Pi_{F_{k, l}} \mathbf{R}_{k-1}\right\|_{F}^{2}$.

In this case, simple algebraic manipulations show that the orthogonal projection of a matrix $\mathbf{M}$ in the space $F_{S}$ spanned by the rank-1 terms $\mathbf{a}_{l} \mathbf{a}_{m}^{H}$ for $l, m \in S$ is

$$
\Pi_{F_{S}}(\mathbf{M})=\mathbf{A}_{S}^{\perp} \mathbf{A}_{S}^{\perp}{ }^{H} \mathbf{M} \mathbf{A}_{S}^{\perp} \mathbf{A}_{S}^{\perp H}
$$

where $\mathbf{A}_{S}^{\perp}$ is an orthogonal basis for the space spanned by the $\mathbf{a}_{l}, l \in S$. The selection criterion at iteration $k$ is:

$$
\begin{aligned}
\rho_{k, l} & =\left\|\Pi_{F_{k, l}}(\mathbf{G})\right\|_{F}^{2} \\
& =\left\|\mathbf{A}_{S_{k, l}}^{\perp} \mathbf{A}_{S_{k, l}}^{\perp} \mathbf{G} \mathbf{A}_{S_{k, l}}^{\perp} \mathbf{A}_{S_{k, l}}^{\perp H}\right\|_{F}^{2} \\
& =\left\|\mathbf{A}_{S_{k, l}}^{\perp} \mathbf{G} \mathbf{A}_{S_{k, l}}^{\perp}\right\|_{F}^{2}
\end{aligned}
$$

The CMF-OLS algorithm for covariance matrix estimation then writes:

1. Initialization: $k=1$, residual $\mathbf{R}_{0}=\mathbf{G}$, set of indices $S_{0}=\emptyset$

2. Projections of the residual on the blocks are computed, $\rho_{k, l}=\left\|\Pi_{F_{k, l}}\left(\mathbf{R}_{k-1}\right)\right\|_{F}^{2}$

3. An additional source is identified by the maximal norm of the projection $l^{\star}=\operatorname{argmax}_{l} \rho_{k, l}$ 
4. Its index is added to the set $S_{k+1}=S_{k} \cup\left\{l^{\star}\right\}$

5 . The residual is updated by orthogonaly projecting $\mathbf{G}$ on the space spanned by the identified atoms: $\mathbf{R}_{k}=\mathbf{G}-\Pi_{F_{k+1}}(\mathbf{G})$

6. $k=k+1$ and go to step 2 until a stopping criterion is met.

After $K$ iterations, the OLS estimation of the $K \times K$ covariance matrix $\tilde{\mathbf{C}}$ is given by the solution of the leastsquares problem

$$
\hat{\tilde{\mathbf{C}}}_{O} L S=\underset{\mathbf{C}}{\operatorname{argmin}}\left\|\mathbf{G}-\mathbf{A}_{S_{K}} \mathbf{C A}_{S_{K}}^{H}\right\|_{F}^{2} .
$$

with explicit solution $\hat{\tilde{\mathbf{C}}}_{O L S}=\mathbf{A}_{S_{K}}^{\dagger} \mathbf{G A}_{S_{K}}^{\dagger H}$. where $\cdot^{\dagger}$ denotes the Moore-Penrose pseudo-inverse. The estimate $\hat{\mathbf{C}}_{O L S}$ of the complete covariance matrix $\mathbf{C}$ is then given by setting the diagonal and off-diagonal coefficients with indices in $S_{K}$ with the coefficients of $\hat{\tilde{\mathbf{C}}}_{O L S}$.

The main computational burden is the computation of the selection criterion. This criterion can be efficiently computed the following way. The orthogonal matrix $\mathbf{A}_{S_{k, l}}^{\perp}$ is obtained by concatenating $\mathbf{A}_{S_{k}}^{\perp}$ with the normalized orthogonal projection $\tilde{\mathbf{a}}_{l}$ of $\mathbf{a}_{l}$ on the orthogonal of $\mathbf{A}_{S_{k}}^{\perp}$. The criterion then writes

$$
\begin{aligned}
\left\|\mathbf{A}_{S_{k, l}}^{\perp H} \mathbf{G A}_{S_{k, l}}^{\perp}\right\|_{F}^{2}= & \left\|\left(\begin{array}{c}
\mathbf{A}_{S_{k-1} H}^{\perp H} \\
\tilde{\mathbf{a}}_{l}^{H}
\end{array}\right) \mathbf{G}\left(\mathbf{A}_{S_{k-1}}^{\perp} \tilde{\mathbf{a}}_{l}\right)\right\|_{F}^{2} \\
= & \left\|\mathbf{A}_{S_{k-1}}^{\perp H} \mathbf{G} \mathbf{A}_{S_{k-1}}^{\perp}\right\|_{F}^{2}+ \\
& 2\left\|\tilde{\mathbf{a}}_{l}^{H} \mathbf{G} \mathbf{A}_{S_{k-1}}^{\perp}\right\|_{2}^{2}+\left|\tilde{\mathbf{a}}_{l}^{H} \mathbf{G} \tilde{\mathbf{a}}_{l}\right|^{2} .
\end{aligned}
$$

The first term being constant with respect to $l$, only the last two terms have to be computed.

\section{B. Computational complexity}

The computational complexity of the algorithm depends on the number of sensors $N$, the discretization size $L$ and the number of iterations $K$, that are such that $K \leq N \leq L$. The computational complexity of an iteration of OLS is governed by

- the construction of the orthogonal matrices $A_{S_{k, l}}^{\perp}$, by projection and normalization of the $\mathbf{a}_{l}$ : $O(L K N)$

- and the computation of the norm of the projection using the two last terms of (??): $O\left(L N^{2}\right)$

As $K \leq N$, the total cost is $O\left(L N^{2} K\right)$. We note that the complexity is linear with respect to the size of the grid $L$. The memory usage of CMF-OLS is dominated by the size of the dictionary $L N$.

As a comparison, MACS has a time complexity in $L^{3}$, and the time complexity of DAS-C is $O\left(L^{2} N\right)$, quadratic in the size of the grid. Memory footprint of MACS and DAS-C is at least the size of the covariance matrix $L^{2}$.

\section{SEPARATION OF THE SOURCES}

Once the covariance matrix is estimated, the different sets of correlated sources can be identified. First, the number of groups of correlated sources is estimated by computing the eigenvalues of the covariance matrix. The number of groups $G$ is the number of singular values higher than the noise floor.

The information on their spatial shape is included in the singular vectors of the estimated covariance matrix. The singular vector associated to a group describe the relative level of the sources at their respective locations. Combined with the corresponding singular values, respective powers of the sources can be estimated in each group.

However, because of the presence of noise, the singular vectors are combinations of several spatial shapes for sources associated with similar eigenvalues. A simple algorithm is proposed to disentangle the sources, based on the assumption that the supports of the sources are disjoint. With $\mathbf{v}_{k}$ the first $K$ singular vectors of $\hat{\tilde{\mathbf{C}}}_{O L S}$, assumed to be normalized, and $v_{k l}$ their coefficients, the source shapes $\mathbf{s}_{k}$ are estimated by the following algorithm:

\section{1. $k=1$}

2. Compute $l^{\star}=\operatorname{argmax}_{l}\left|\sum_{n=1}^{K} v_{n l}\right|^{2}$,

3. Let $\mathbf{s}_{k}=\sum_{n=1}^{K} \bar{v}_{n l^{\star}} \mathbf{v}_{n} / \sqrt{\sum_{n=1}^{K}\left|v_{n l^{\star}}\right|^{2}}$,

4. Replace the $K$ vector $\mathbf{v}_{n}$ by their projections on the orthogonal of $\mathbf{s}_{k}$.

5. $k=k+1$ and go to step 2 until $k=G$.

The algorithm is supported by the following interpretation: the vectors $\mathbf{v}_{k}$ form an orthogonal basis of the space spanned by the shape vectors $\mathbf{s}_{k}$, itself an orthogonal basis as their support are disjoint. Each step of the algorithm rotates the basis $\mathbf{v}_{k}$ such that one of the vector is equal to one of the shape vectors. The algorithm is then iterated in the space orthogonal to this vector.

The estimated covariance matrix is then projected on the rank 1 matrices $\mathbf{s}_{k} \mathbf{s}_{k}^{H}$, with coefficients $\alpha_{k}$. The final estimation of the covariance matrix is:

$$
\hat{\tilde{\mathbf{C}}}=\sum_{i=1}^{G} \alpha_{i} \mathbf{s}_{i} \mathbf{s}_{i}^{H} .
$$

From this decomposition, one can

- compute the power emitted at each location of space: $\hat{P}_{l}=\sum_{k=1}^{G} \alpha_{k}\left|s_{k l}\right|^{2}$,

- or analyze a unique source by using its rank-one description $\alpha_{k} \mathbf{s}_{k} \mathbf{s}_{k}^{H}$.

The computational complexity of this step is dominated by the SVD of the matrix $\hat{\tilde{\mathbf{C}}}_{O L S}$, in $O\left(K^{3}\right)$, itself dominated by the complexity of the OLS algorithm. 


\section{SIMULATIONS}

The method is demonstrated on a simple case by simulating a linear antenna, and locating correlated sources on a line parallel to the array at a distance of $5 \mathrm{~m}$. The antenna is composed of $N=19$ sensors spaced by a half wavelength. Two groups of coherent sources are considered, with respectively 2 and 3 narrow band sources emitting at $3500 \mathrm{~Hz}$. Locations and powers of the sources are given in table ??. The SNR is set to $0 \mathrm{~dB}$ and $I=500$ time samples are collected. The $1 \mathrm{D}$ region of interest of the sources is discretized with a $5 \mathrm{~mm}$ step, such that $L=400$ grid points are considered.

The estimation of the source covariance matrix provided by the DAS-C beamformer is pictured on figure ?? Its diagonal, the output of the standard DAS beamformer, is plotted on figure ??. We note in particular that the two less powerful sources cannot be identified by DAS beamforming, as they are below the level of the sidelobes of more powerful sources. Correlations between sources are represented by the off-diagonal coefficients.

The coefficients of the covariance matrix estimated by CMF-OLS are pictured on figure ??. One can note that the two groups of correlated sources can be easily identified from this estimation while it was not possible with the DAS-C estimation. The estimated positions and powers of the sources estimated by CMF-OLS are given in table ??, and plotted on figure ??. Positions and powers of the five sources are estimated correctly, even for the sources that cannot be identified by beamforming.

The source powers estimated by IMACS are plotted on fig ??. As the value proposed for the regularization parameter in (?) did not yield accurate results, it was set manually. The rank of the covariance matrix is assumed to be $L_{h}=2$, and 50 iterations are used. The weaker sources are not estimated accurately by IMACS. Moreover, for some sources, the power is spread over contiguous grid points, making the estimation of the power difficult. This is a well known phenomenon in sparse deconvolution (?), that cannot be avoided by refining the discretization.

The energy of the residual before each step of CMFOLS is plotted on figure ??. The number of sources is estimated by the location of the discontinuity in the decay of the energy, here at $K=5$ iterations. The singular values of the estimated covariance matrix with 5 iterations are plotted on the same figure, showing that the sources can be separated in two correlated groups.

Computation times for CMF-OLS is $0.003 \mathrm{~s}$, and $22.7 \mathrm{~s}$ for IMACS. For this set of data, the proposed methods is about 10000 times faster that IMACS algorithm which is known as one of the fastest approaches for source covariance matrix estimation. The algorithm is implemented in MATLAB R2018b, and is run on a laptop equipped with an Intel Core i7-7820HQ CPU @ $2.90 \mathrm{GHz}$ $\times 8 \mathrm{CPU}$ and $16 \mathrm{~GB}$ memory.

\begin{tabular}{|l|ccccc|}
\hline \multicolumn{7}{|c|}{ True values } \\
\hline Positions [m] & -0.8 & 0.0 & 0.9 & -0.3 & 0.5 \\
Power [dB] - group 1 & -7.95 & 0.00 & -13.98 & - Inf & - Inf \\
Power [dB] - group 2 & - Inf & - Inf & - Inf & -10.46 & -1.94 \\
\hline \hline \multicolumn{7}{|c|}{ Estimation by CMF-OLS } \\
\hline Positions [m] & -0.79 & 0.00 & 0.89 & -0.30 & 0.50 \\
Power [dB] - group 1 & -7.78 & 0.07 & -13.59 & -36.70 & -334.59 \\
Power [dB] - group 2 & -32.52 & -37.16 & -27.10 & -10.32 & -1.55 \\
\hline
\end{tabular}

TABLE I. Simulation (5 sources). Position and Power of the sources: true values and estimations by CMF-OLS. Powers are given with reference to the most powerful source at $x=$ 0.0 .
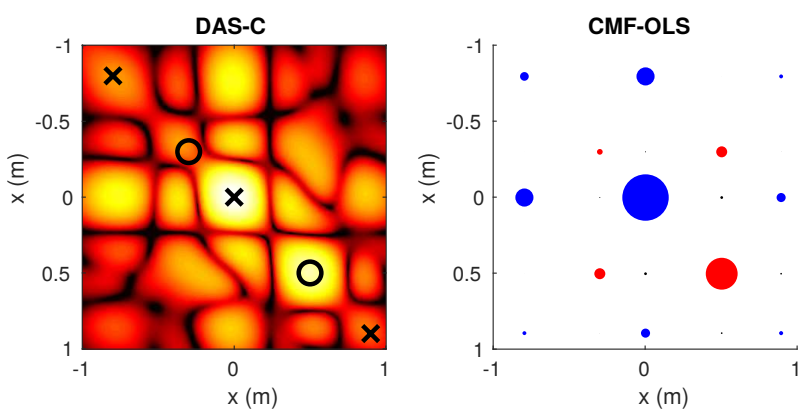

FIG. 2. Simulations (5 sources) - source covariance matrix. In both representation, the powers of the sources are found on the diagonal, off-diagonal terms indicate correlation between sources.

Left: Output of DAS-C, the actual sources are superimposed, crosses and circles denotes the two source groups.

Right: Covariance matrix estimated by CMF-OLS, the radius of the disks are proportional to the absolute value of the coefficients.

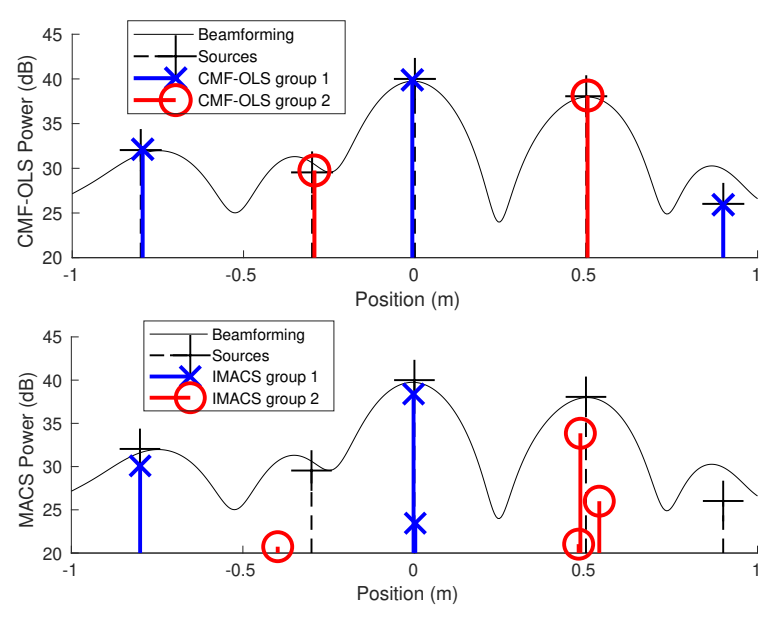

FIG. 3. Simulations (5 sources) - position and power.

Top: estimation by CMF-OLS, $K=5$.

Bottom: estimation by IMACS. 

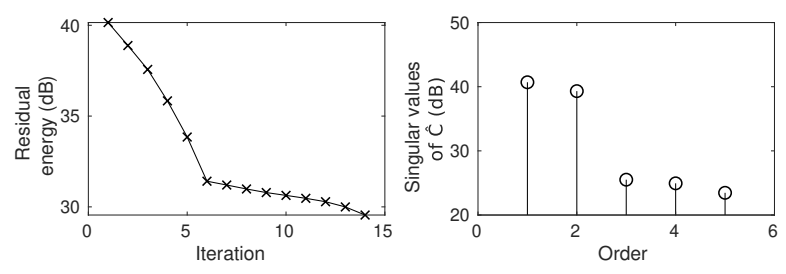

FIG. 4. Simulations (5 sources).

Left: Energy of the residual of the CMF-OLS.

Right: Singular values of the covariance matrix.

\section{EXPERIMENTAL VALIDATION}

The method is now tested experimentally in an anechoic chamber. The following experiments, make use of the same sources built out of baffled broadband omnidirectional loudspeakers (Visaton-BF32 - [150Hz-20kHz]).

\section{A. Set Up}

Two different acoustic antennas are implemented. They use the same acoustic sensors : MEMS digital microphones (invensense - INMP441) with a 26 dBFS sensitivity $(1 \mathrm{kHz}, 94 \mathrm{dBSPL})$ and a flat response in the band [150 Hz - $15 \mathrm{kHz}]$.

The first antenna counts 32 elements irregularly spaced on a straight line, $1.36 \mathrm{~m}$ long, with an average step of $45 \mathrm{~mm}$.

The second antenna counts 128 elements distributed along 16 linear rays. The 8 microphones on a ray are spaced according to a geometric law and the origins of the 16 rays follow a pseudo random distribution.

The second microphone array and the sources are pictured on fig. ??. They are located in two parallel planes, at a distance $d=4.3 \mathrm{~m}$.

The microphone signals are sampled at $F s=50 \mathrm{kHz}$ and analyzed by Short-Term Fourier Transform, with a 2048 samples Hann window (41ms duration and $75 \%$ overlap).

\section{B. Experiment 1: Linear array}

The linear array is used in this experiment. Four sources are located at a $5.18 \mathrm{~m}$ distance. They are correlated by pairs. The source space consists of a parallel $2 \mathrm{~m}$ long straight line with a $5 \mathrm{~mm}$ step ( $L=400$ points).

The output of the DAS beamforming is pictured on figure ?? at frequency $F=11 \mathrm{kHz}$. The power of the weaker source is below level of a more powerful source, and cannot be located. On the same figure, crosses and circles indicates the sources found by CMF-OLS. They are correctly grouped, and their location is close to the actual locations of the source (dashed lines). The number of iterations, $K=5$, and the number of groups, $J=2$ are obtained form the decay of the residual energy and the singular values of the covariance matrix.
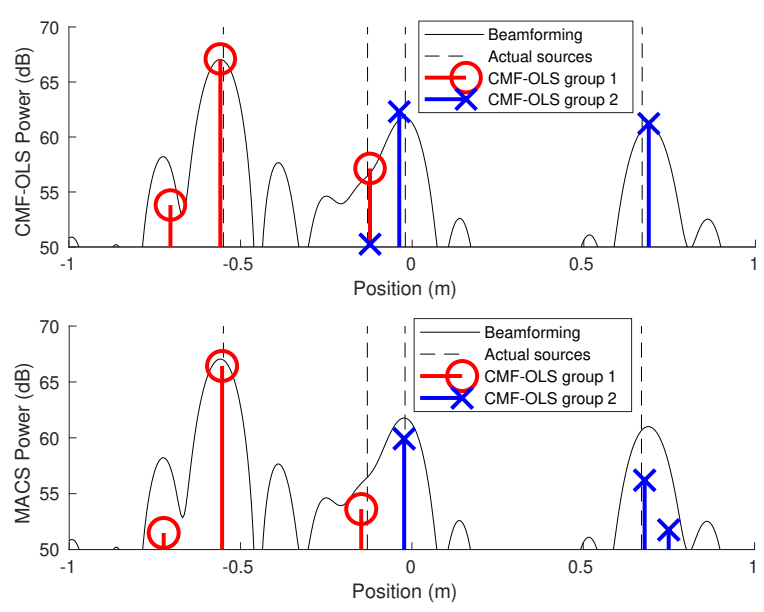

FIG. 5. Experimental results, linear array. Top: Two pairs of correlated $F=2.2 \mathrm{kHz}$. The sources identified by CMFOLS are superimposed over the output of the standard beamformer. Bottom: Estimation by IMACS.
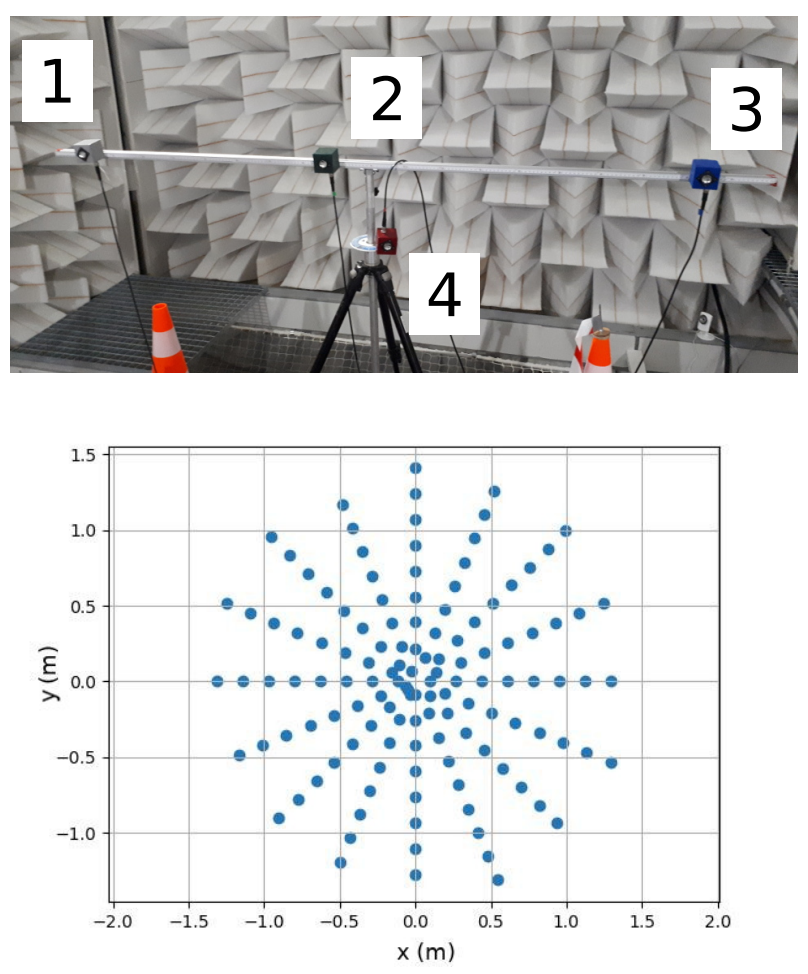

FIG. 6. Experimental setup. Top: Acoustical sources. Bottom: Positions of the array microphones.

The bottom part of the figure shows the source estimated by IMACS. Similar results to CMF-OLS are obtained, with a computation time of $24.3 \mathrm{~s}$, compared to 0.06s for CMF-OLS. 


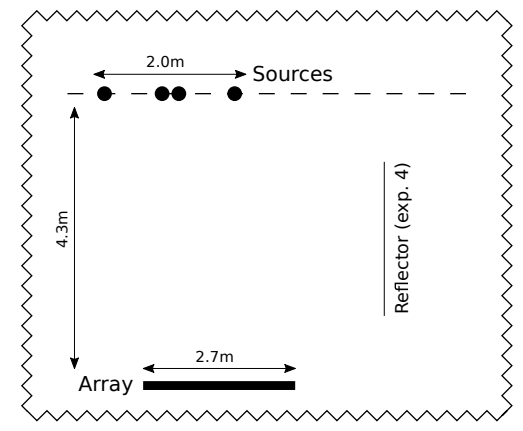

FIG. 7. Experimental setup

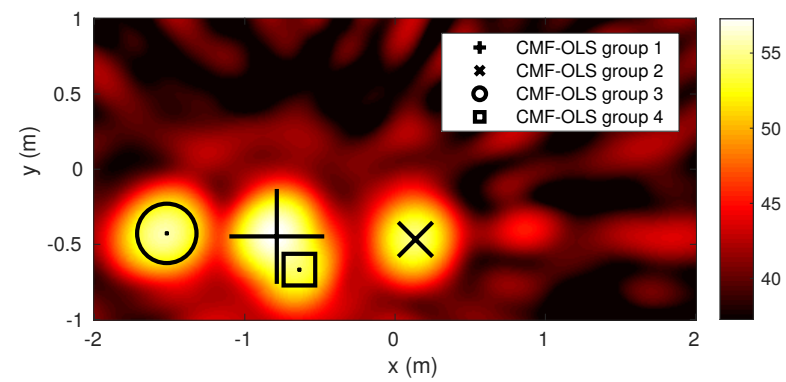

FIG. 8. Experimental results with four uncorrelated sources at $F=2.2 \mathrm{kHz}$. The sources identified by CMF-OLS are superimposed over the output of the standard beamformer.

\section{Experiment 2: 2D array, Uncorrelated sources}

The localization of uncorrelated sources is tested first. They sources are located in a parallel plane, at a distance $d=4.3 \mathrm{~m}$. The region of interest is a $2 \mathrm{~m} \times 4 \mathrm{~m}$ rectangle discretized over $M=400 \times 200=8 \mathrm{e} 4$ points.

The output of the DAS beamforming is pictured on figure ?? at frequency $F=2.2 \mathrm{kHz}$. Source 4 cannot be identified as it is below the level of the mainlobe of source 2 .

The sources identified by CMF-OLS are superimposed on the same image. The number of iterations $(K=4)$ and number of source groups 4$)$ are chosen according to the residual energy decay and the singular values of the estimated covariance matrix, see figure ??.

The computation time is here 1.4s. As the complexity of the MACS method is proportional to $L^{3}$, computation times for MACS would be in the order of several years. The size of the output of DAS-C is $L^{2}$, more than $25 \mathrm{~GB}$ as single floats, larger than the memory of the computer used for the numerical applications.

\section{Experiment 3: 2D array, Pairs of correlated sources}

In the next experiment, the same setup is used, with two pairs of correlated sources (1-2, 3-4). As in the previous experiment, source 4 cannot be identified by DAS beamforming.
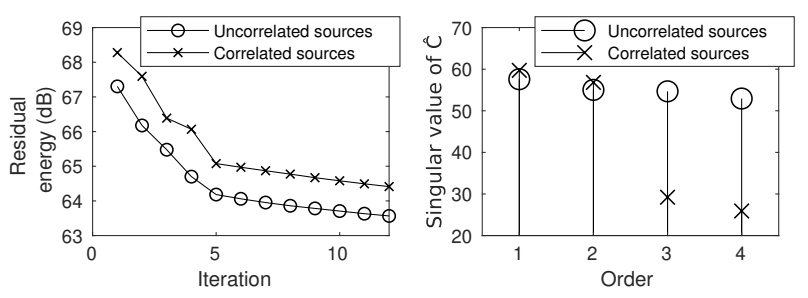

FIG. 9. Experimental results with uncorrelated (Exp. 2) and correlated sources (Exp. 3). Left: Energy of the residual before each step of the CMF-OLS algorithm. Right: Singular values of the estimated covariance matrix for $K=4$.

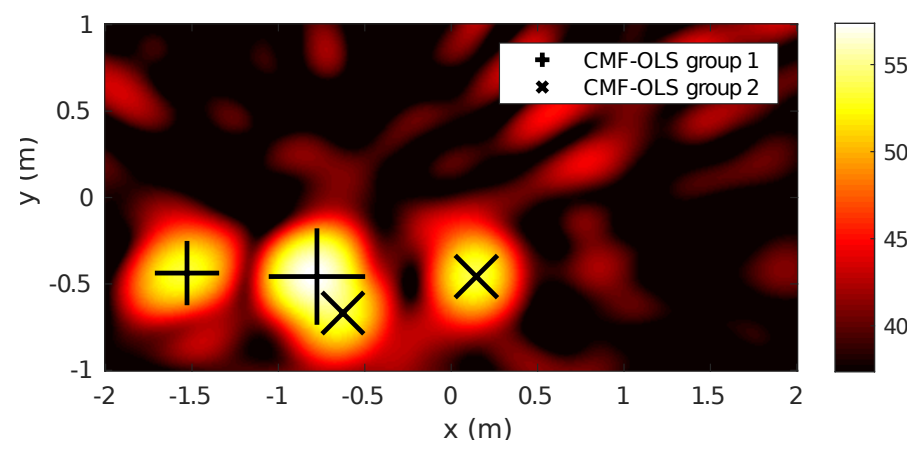

FIG. 10. Experimental results with two pairs of correlated sources at $F=2.2 \mathrm{kHz}$. The sources identified by CMFOLS are superimposed over the output of the standard beamformer.

For CMF-OLS, the residual energy decay indicates four sources as above. Here, two groups are identified using the singular values of the estimated covariance matrix, superimposed on the output of the beamformer on figure ??.

The estimated covariances with individual sources are pictured on figure ??. The covariances are given by the column of the estimated covariance matrix $\tilde{\mathbf{C}}$ associated to a given source. In the case of source 1 , both DAS-C and OMF-CLS yield correct estimation of the covariances, as source 1 is correlated with source 2 only. The covariances estimated by DAS-C for source 4 are however inexact, as source 4 is estimated to be correlated with all four sources. This is explained by the fact that source 4 is in the main lobe of source 3, itself correlated with source 1. CMF-OLS does not find significant correlation with source 3 only.

\section{E. Experiment 4: 2D array, Sources with reflections}

Finally, the identification of reflections is tested. A reflector is set up in the anechoic room, and uncorrelated sources (3 and 4) are used. The sources and their reflections are expected to be strongly correlated, and the number of correlated blocks is the number of actual sources. The scan area is augmented to include points in the reflected, virtual space, with dimension $7 \mathrm{~m} \times 1.5 \mathrm{~m}$, 

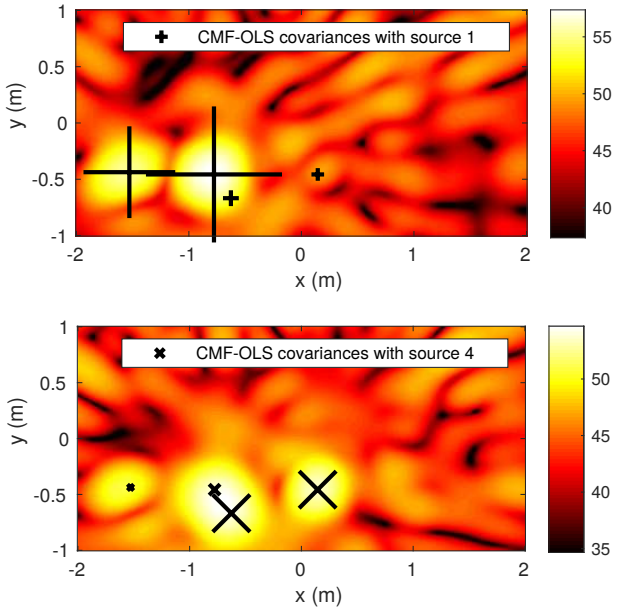

FIG. 11. Experimental results. Covariances estimated by DAS-C and CMF-OLS with a source. Top: source 4 , bottom: source 1.

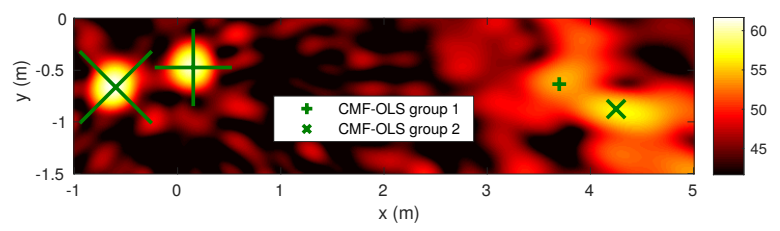

FIG. 12. Experimental results with two sources and their reflections at $F=2.93 \mathrm{kHz}$. The sources identified by CMFOLS are superimposed over the output of the standard beamformer.

and $L=1.05 \mathrm{e} 5$ points. The computation time is here $1.74 \mathrm{~s}$. The four sources located by OLS are correctly identified as two pairs of correlated sources, one real source and its reflection.

\section{CONCLUSION}

In this paper the CMF-OLS method is proposed for the estimation of the source covariance matrix which provides simultaneously location, power, and mutual covariances of acoustic sources. The interest of this approach compared to standard methods is its ability to distinguish correlated sources. Simulations and experimental results are presented that illustrate this capacity.

The proposed method is a greedy approach based on the OLS algorithm. Its advantage lies in a very low numerical complexity which is linear with respect to the number of grid points in the scanned source domain. Consequently it is drastically faster than similar methods based on the optimization of a regularized criterion. Numerical examples show that the computing time is divided by a factor 1000 at least. To our knowledge, it is the first method for source covariance estimation that can deal with large numbers of grid points, making it usable in a wide range of applications. An other advantage of the CMF-OLS method upon the regularization approach, is that it does not require the tuning of any parameter to get the optimal solution.

Finally experimental results in free field and semi free field were presented which prove that the accuracy of CMF-OLS outperforms those of regularized approaches such as the MACS or IMACS methods, in terms both of localization and power estimation.

H. Krim and M. Viberg, "Two decades of array signal processing research: the parametric approach," IEEE Signal Processing Magazine 13(4), 67-94 (1996) doi: 10.1109/79.526899.

J. Antoni, T. L. Magueresse, Q. Leclère, and P. Simard, "Sparse acoustical holography from iterated bayesian focusing," Journal of Sound and Vibration 446, 289 325 (2019) http://www.sciencedirect.com/science/article/ pii/S0022460X1930001X doi: https://doi.org/10.1016/j.jsv. 2019.01.001.

N. Chu, A. Mohammad-Djafari, and J. Picheral, "Robust Bayesian super-resolution approach via sparsity enforcing a priori for near-field aeroacoustic source imaging," Journal of Sound and Vibration 332(18), 4369-4389 (2013) https://hal. archives-ouvertes.fr/hal-00794230.

A. Xenaki, P. Gerstoft, and K. Mosegaard, "Compressive beamforming," The Journal of the Acoustical Society of America 136(1), 260-271 (2014) http://asa.scitation.org/doi/10. 1121/1.4883360 doi: 10.1121/1.4883360.

E. Fernandez-Grande and L. Daudet, "Compressive acoustic holography with block-sparse regularization," The Journal of the Acoustical Society of America 143(6), 3737-3746 (2018) http: //asa.scitation.org/doi/full/10.1121/1.5042412 doi: 10. 1121/1.5042412.

G. Chardon, L. Daudet, A. Peillot, F. Ollivier, N. Bertin, and R. Gribonval, "Near-field acoustic holography using sparse regularization and compressive sampling principles," The Journal of the Acoustical Society of America 132(3), 1521-1534 (2012) https://doi.org/10.1121/1.4740476 doi: 10.1121/1.4740476.

D. Malioutov, M. Cetin, and A. S. Willsky, "A sparse signal reconstruction perspective for source localization with sensor arrays," IEEE Transactions on Signal Processing 53(8), 3010-3022 (2005) doi: 10.1109/TSP.2005.850882.

A. Das, W. S. Hodgkiss, and P. Gerstoft, "Coherent Multipath Direction-of-Arrival Resolution Using Compressed Sensing," IEEE Journal of Oceanic Engineering 42(2), 494-505 (2017) doi: 10.1109/JOE. 2016.2576198.

J. Zheng and M. Kaveh, "Sparse Spatial Spectral Estimation: A Covariance Fitting Algorithm, Performance and Regularization," IEEE Transactions on Signal Processing 61(11), 27672777 (2013) doi: 10.1109/TSP. 2013.2256903.

T. Yardibi, J. Li, P. Stoica, N. S. Zawodny, and L. N. Cattafesta, "A covariance fitting approach for correlated acoustic source mapping," The Journal of the Acoustical Society of America 127(5), 2920-2931 (2010) https://doi.org/10.1121/1.3365260 doi: $10.1121 / 1.3365260$.

S. Chen, S. A. Billings, and W. Luo, "Orthogonal least squares methods and their application to non-linear system identification," International Journal of Control 50(5), 1873-1896 (1989) https://doi.org/10.1080/00207178908953472 doi: 10. 1080/00207178908953472.

Y. C. Pati, R. Rezaiifar, Y. C. P. R. Rezaiifar, and P. S. Krishnaprasad, "Orthogonal matching pursuit: Recursive function approximation with applications to wavelet decomposition," in Proceedings of the 27 th Annual Asilomar Conference on Signals, Systems, and Computers (1993), pp. 40-44.

C. Soussen, R. Gribonval, J. Idier, and C. Herzet, "Joint KStep Analysis of Orthogonal Matching Pursuit and Orthogonal Least Squares," IEEE Transactions on Information Theory 59(5), 3158-3174 (2013) doi: 10.1109/TIT.2013.2238606. 
J. W. Paik, W. Hong, J.-K. Ahn, and J.-H. Lee, "Statistics on noise covariance matrix for covariance fitting-based compressive sensing direction-of-arrival estimation algorithm: For use with optimization via regularization," The Journal of the Acoustical Society of America 143(6), 3883-3890 (2018) http://asa. scitation.org/doi/full/10.1121/1.5042354 doi: 10.1121/1. 5042354.

T. Yardibi, J. Li, P. Stoica, and L. N. Cattafesta, "Sparsity constrained deconvolution approaches for acoustic source mapping," The Journal of the Acoustical Society of America 123(5), 26312642 (2008) https://doi.org/10.1121/1.2896754 doi: 10.1121/ 1.2896754.

W. Xiong, J. Picheral, S. Marcos, and G. Chardon, "Sparsitybased localization of spatially coherent distributed sources," in 2016 IEEE International Conference on Acoustics, Speech and Signal Processing (ICASSP), Shangai, China (2016), https: //hal-supelec.archives-ouvertes.fr/hal-01231789, doi: 10. 1109/ICASSP. 2016.7472276.

M. Grant and S. Boyd, "CVX: Matlab software for disciplined convex programming, version 2.1," http://cvxr.com/cvx (2014).

M. Grant and S. Boyd, "Graph implementations for nonsmooth convex programs," in Recent Advances in Learning and Control, edited by V. Blondel, S. Boyd, and H. Kimura, Lecture Notes in Control and Information Sciences (Springer-Verlag Limited, 2008), pp. 95-110, http://stanford.edu/ boyd/graph_ dcp.html.

Y. Li, M. Li, D. Yang, and C. Gao, "Research of the improved mapping of acoustic correlated sources method," Applied Acoustics 145, 290-304 (2019) http://www.sciencedirect. com/science/article/pii/S0003682X17309118 doi: 10.1016/j. apacoust.2018.10.009.

A. Peillot, F. Ollivier, G. Chardon, and L. Daudet, "Localization and identification of sound sources using "compressive sampling" techniques," in 18th International Congress on Sound and Vibration, Rio de Janeiro, Brazil (2011), https://hal.inria.fr/ hal-00766968.

T. Padois and A. Berry, "Orthogonal matching pursuit applied to the deconvolution approach for the mapping of acoustic sources inverse problem," The Journal of the Acoustical Society of America 138(6), 3678-3685 (2015) https://doi.org/10.1121/ 1.4937609 doi: 10.1121/1.4937609.

Y. C. Eldar, P. Kuppinger, and H. Bolcskei, "Block-Sparse Signals: Uncertainty Relations and Efficient Recovery," IEEE Transactions on Signal Processing 58(6), 3042-3054 (2010) doi: 10. 1109/TSP. 2010. 2044837.

G. Chardon, "A block-sparse MUSIC algorithm for the localization and the identification of directive sources," in 2014 IEEE International Conference on Acoustics, Speech and Signal Processing (ICASSP) (2014), pp. 3953-3957, doi: 10.1109/ICASSP. 2014. 6854343.

M. R. Bai, C. Chung, and S.-S. Lan, "Iterative algorithm for solving acoustic source characterization problems under block sparsity constraints," The Journal of the Acoustical Society of America 143(6), 3747-3757 (2018) http://asa.scitation.org/doi/ full/10.1121/1.5042221 doi: 10.1121/1.5042221.

V. Duval and G. Peyré, "Sparse regularization on thin grids I: the Lasso," Inverse Problems 33(5), 055008 (2017) doi: 10.1088/ 1361-6420/aa5e12. 